\title{
Local Spectral Properties Under Conjugations
}

\author{
Pietro Aiena®, Fabio Burderi and Salvatore Triolo
}

\begin{abstract}
In this paper, we study some local spectral properties of operators having form $J T J$, where $J$ is a conjugation on a Hilbert space $H$ and $T \in L(H)$. We also study the relationship between the quasinilpotent part of the adjoint $T^{*}$ and the analytic core $K(T)$ in the case of decomposable complex symmetric operators. In the last part we consider Weyl type theorems for triangular operator matrices for which one of the entries has form $J T J$, or has form $J T^{*} J$. The theory is exemplified in some concrete cases.
\end{abstract}

Mathematics Subject Classification. Primary 47B35; Secondary 47A10, 47A11.

Keywords. Conjugations, Local spectral properties, Weyl-type theorems for upper triangular operator matrices.

\section{Introduction}

In this paper we will continue the analysis undertaken in $[15,16]$ on the general problem of studying the local spectral properties of complex symmetric operators on separable Hilbert spaces $H$. Recall that a conjugation on $H$ is conjugate-linear operator $J: H \rightarrow H$ which satisfies

$$
\langle J x, J y\rangle=\langle y, x\rangle \quad \text { for all } x, y \in H
$$

and $J$ is involutive, i.e., $J^{2}=I$. Evidently, $J$ is isometric and $T^{J}:=J T J \in$ $L(H)$ for every $T \in L(H)$. If $T^{*}$ denote the Hilbert adjoint of $T \in L(H)$, we say that $T$ is $J$-complex symmetric, with respect to the conjugation $J$, if $T^{J}=T^{*}$, i.e., $T=J T^{*} J$, or equivalently, $J T=T^{*} J$, or $J T^{*}=T J$. If $T$ is $J$-symmetric for some conjugation $J$ we say simply that $T$ is complex symmetric. The terminology is due to fact that $T$ is complex symmetric exactly when $T$ is unitarily equivalent to a symmetric matrix with complex entries, regarded as an operator acting on an $l^{2}$-space of appropriate dimension, cf. [12]. The class of symmetric complex operators is rather large and includes some important classes of operators, for instance normal operators, 
Hankel operators, compressed Toeplitz operators on Hardy spaces, the classical Volterra integration operator, every idempotent operator, and nilpotent operator of order two, see $[13]$. In $[15,16]$ the authors have investigated some aspects of local spectral theory for complex symmetric operators, for instance the transmission from $T$ to $T^{*}$ of Dunford property $(C)$, property $(\delta)$, property $(\beta)$, and decomposability. In this paper we consider the transmission of these properties from $T$ to $T^{J}$, where $J$ is any conjugation. In particular we extend some results obtained in [17].

Other results concern the relationship between the quasi-nilpotent part $H_{0}\left(T^{*}\right)$ of $T^{*}$ and the analytic core $K(T)$ in the case of decomposable operators, in general, and in the particular case where $T$ is complex symmetric decomposable operator.

In the last part we give some other results, concerning Weyl type theorems in the framework of operator matrices. Weyl type theorems for operator matrices have been studied by several authors, see for instance Duggal [9]. In this paper we study Weyl type theorems for $f(T)$, where $f$ is an analytic function defined on an open neighbourhood of the spectrum, in the case that the upper triangular operator matrix $T$ has an entry of the form $T^{J}$, where $J$ is a conjugation. Such operator matrices have been also investigated by Jung et al. [17], and some of the results here obtained may be thought as complementing their results.

\section{Local Spectral Theory and Conjugations}

Throughout this paper $H$ denotes a separable complex Hilbert space and, given a subset $F \subseteq \mathbb{C}$, by $\bar{F}$ we always denote the set $\{\bar{z}: z \in F\}$. Let $J$ be any conjugation on $H$. It is easily seen that $J T^{J} J=J J T J J=T$ and

$$
T=T^{J} \Leftrightarrow T J=J T,
$$

so, if $T$ is $J$-complex symmetric then

$$
T \text { is selfadjoint } \Leftrightarrow T J=J T .
$$

The operator $T^{* J}=J T^{*} J$ has been called the $J$-transpose of $T$ by some authors. For every $x, y \in H$ we have;

$$
\langle J T J x, y\rangle=\left\langle J T J x, J^{2} y\right\rangle=\langle J y, T J x\rangle=\left\langle T^{*} J y, J x\right\rangle=\left\langle J^{2} T^{*} J y, J x\right\rangle=\left\langle x, J T^{*} J y\right\rangle,
$$

so $\left(T^{J}\right)^{*}=(J T J)^{*}=J T^{*} J=T^{*} J$. It is easily seen that $\sigma\left(T^{J}\right)=\overline{\sigma(T)}$, from which we conclude that if $T J=J T$ then $\sigma(T) \subset \mathbb{R}$. Moreover,

$$
T T^{J}=T^{J} T \Leftrightarrow(T J)^{2}=(J T)^{2} .
$$

The following property, the single-valued extension property, which dates back to the early days of local spectral theory, has been introduced by Dunford $[10,11]$. In the sequel we consider the following localized version of this property.

Definition 2.1. An operator $T \in L(X), X$ a Banach space, is said to have the single valued extension property at $\lambda_{0} \in \mathbb{C}$, in short $T$ has the SVEP 
at $\lambda_{0}$, if for every open disc $\mathbf{D}_{\lambda_{0}}$ centered at $\lambda_{0}$ the only analytic function $f: \mathbf{D}_{\lambda_{0}} \rightarrow H$ which satisfies the equation

$$
(\lambda I-T) f(\lambda)=0
$$

is the constant function $f \equiv 0 . T$ is said to have the SVEP if $T$ has the SVEP for every $\lambda \in \mathbb{C}$.

The SVEP has a crucial role in local spectral theory consequently, it has a certain interest to find conditions for which an operator has, or does not have, the SVEP. Observe first that in general the SVEP from an operator $T$ on a Banach space is not transmitted to the dual $T^{\prime}$, or to the adjoint $T^{*}$ in the case of Hilbert space operators. Furthermore, if $T \in L(H), T^{\prime}$ has SVEP at $\lambda$ if and only if $T^{*}$ has SVEP at $\bar{\lambda}$.

For instance the unilateral right shift $R$ on $l^{2}(\mathbb{N})$ has SVEP while its adjoint $L$, which coincides with the left unilateral shift, does not satisfies SVEP, see [2, page 135]. A complex symmetric operators may fail SVEP. For instance, if $T:=R \oplus L$ then $T$ is complex symmetric on $l^{2} \oplus l^{2}$ by [24], but $T$ does not satisfy SVEP by [2, Theorem 2.15]. However we have:

Theorem 2.2. Let $T \in L(H)$ and let $J$ be any conjugation. Then the following statements are equivalent:

(i) $T$ has $S V E P$ at $\lambda_{0}$;

(ii) $T^{J}$ has $S V E P$ at $\bar{\lambda}_{0}$.

Proof. (i) $\Rightarrow$ (ii). Suppose that $T$ has SVEP at $\lambda_{0}$. Let $\mathbb{D}_{0}$ be an open disc centered al $\lambda_{0}$, so $\overline{\mathbb{D}_{0}}$ is an open disc centered at $\bar{\lambda}_{0}$. Suppose that $f: \overline{\mathbb{D}}_{0} \rightarrow H$ is an analytic function for which $\left(\bar{\lambda} I-T^{J}\right) f(\bar{\lambda})=(\bar{\lambda} I-J T J) f(\bar{\lambda})=0$ for all $\bar{\lambda} \in \overline{\mathbb{D}_{0}}$. Then

$$
\left.(\bar{\lambda} I-J T J) f(\bar{\lambda})=\left(\bar{\lambda} J^{2}-J T J\right) f \overline{(} \lambda\right)=J(\lambda I-T) J f(\bar{\lambda})=0 .
$$

Since $J$ is injective then $(\bar{\lambda} J-T) J f(\bar{\lambda})=0$ for $\bar{\lambda} \in \overline{\mathbb{D}_{0}}$. Define $h(\lambda):=J f(\bar{\lambda})$ for $\lambda \in \mathbb{D}_{0} . h(\lambda)$ is still an analytic function on the disk $\mathbb{D}_{0}$. Indeed, write

$$
f(\bar{\lambda})=\sum_{n=0}^{\infty} a_{n}\left(\bar{\lambda}-\bar{\lambda}_{0}\right)^{n}
$$

then

$$
h(\lambda):=J f(\bar{\lambda})=\sum_{n=0}^{\infty} J a_{n}\left(\bar{\lambda}-\bar{\lambda}_{0}\right)^{n} .
$$

Hence $(\lambda I-T) h(\lambda)=0$ and the SVEP of $T$ at $\lambda_{0}$ entails that $h(\lambda)=J f(\bar{\lambda})=$ 0 , hence $J h(\lambda)=J^{2} f(\bar{\lambda})=f(\bar{\lambda})=0$, so $T^{J}$ has SVEP at $\bar{\lambda}_{0}$.

Conversely, if $T^{J}$ has SVEP at $\bar{\lambda}_{0}$ then, by the first part of the proof, $T=J T^{J} J$ has the SVEP at $\lambda_{0}$.

If $T$ is $J$-complex symmetric we have $T^{*}=J T J$, so we have:

Corollary 2.3. $T \in L(H)$ has $S V E P$ if and only if $T^{J}$ has $S V E P$. If $T \in L(H)$ is complex symmetric, then both operators $T, T^{*}$ have $S V E P$ if one of them has SVEP. 
Hence, for symmetric operators $T$, either $T$ and $T^{*}$ have SVEP, or neither has SVEP. It is known that if for an operator $T \in L(X), X$ a Banach space, both $T$ and $T^{\prime}$ have SVEP then $\sigma(T)=\sigma_{\text {ap }}(T)=\sigma_{\mathrm{s}}(T)$, see $[2$, Theorem 2.68], where $\sigma_{\text {ap }}(T)$ and $\sigma_{\mathrm{s}}(T)$, denote the approximate point spectrum and the surjectivity spectrum, respectively. These equalities hold for any complex symmetric operator, even if it does not have SVEP, see [16]. Corollary 2.3 is very useful to show that an operator $T$ is not complex symmetric. For instance, the right shift $R$ on $\ell^{2}(\mathbb{N})$ has SVEP, but its adjoint, the left shift $L$ does not have SVEP, so $R$ cannot be complex symmetric. Trivially, also every left shift is not complex symmetric, since it has not the SVEP, while its adjoint $R$ has SVEP. Two less trivial cases are given in the sequel.

Let $f \in H^{\infty}(\mathbb{D})$, the Banach algebra of all bounded analytic functions on the open disc $\mathbb{D}$ of $\mathbb{C}$. The multiplication analytic Toeplitz operator $T_{f}$ is defined on the classical Hardy space $H^{2}(\mathbb{D})$ by the assignment $T_{f} g:=f g$ for all $g \in H^{2}(\mathbb{D})$.

Corollary 2.4. Every non-invertible symmetry on a Hilbert space, as well as the multiplication analytic Toeplitz operator on $H^{2}(\mathbb{D})$, is not complex symmetric.

Proof. If $T$ is a non-invertible symmetry then $T$ has SVEP, while SVEP fails fo $T^{\prime}$ at the points $|\lambda|>1$, see [2, Theorem 4.72]. Consequently, $T^{*}$ fails SVEP at the points $|\bar{\lambda}|=|\lambda|>1$. The multiplication Toeplitz operator $T_{f}$ has SVEP, while its dual $T_{f}^{6}$ fails SVEP at the points $\lambda$ which do not belong to the range of $f$, see [2, Theorem 4.75]. Therefore, the adjoint $T_{f}^{*}$ fails SVEP and hence, by Corollary 2.3, $T_{f}$ cannot be complex symmetric. Note that $J T_{f} J$ has SVEP for every conjugation $J$.

For an operator $T \in L(X), X$ a Banach space, we denote by $\sigma_{T}(x)$ the local spectrum of $T$ at the point $x \in X$, see [2] or [20] for definition and properties. An important class subspace in local spectral theory is given by the local spectral subspace associated with a subset $F \subseteq \mathbb{C}$, defined as

$$
X_{T}(F):=\left\{x \in X: \sigma_{T}(x) \subseteq F\right\} .
$$

A variant of the concept of local spectral subspace, which is more useful in the case when $T$ does not have SVEP is defined as follows: if $F$ is a closed subset of $\mathbb{C}$, the glocal subspace $\mathcal{X}_{T}(F)$ is defined as the set of all $x \in X$ for which there exists an analytic function $f: \mathbb{C} \backslash F \rightarrow X$ such that

$$
(\lambda I-T) f(\lambda)=x \quad \text { for all } \lambda \in \mathbb{C} \backslash F .
$$

Note that $\mathcal{X}_{T}(F) \subseteq X_{T}(F)$ for every closed subset $F$ of $\mathbb{C}$, and that $T$ has SVEP if and only if $\mathcal{X}_{T}(F)=X_{T}(F)$ for every closed subset $F$ of $\mathbb{C}$, see $[2$, Theorem 2.23].

In the case of a Hilbert space $H$, for the glocal subspaces $\mathcal{H}_{T}(F)$ we have:

Theorem 2.5. Let $T \in L(H)$ and $J$ any conjugation on $H$.

$$
\mathcal{H}_{T}(F)=J\left(\mathcal{H}_{T^{J}}(\bar{F})\right) \quad \text { and } \quad \mathcal{H}_{T^{J}}(\bar{F})=J\left(\mathcal{H}_{T}(F)\right) \text {, }
$$

for every closed subset $F \subseteq \mathbb{C}$. 
Proof. Let $x \in \mathcal{H}_{T}(F)$. Then there exists an analytic function $f: \mathbb{C} \backslash F \rightarrow H$ such that

$$
(\lambda I-T) f(\lambda)=x, \quad \text { for all } \lambda \notin F .
$$

Set $y:=J x$. Then $J y=J^{2} x=x$. We prove that $y=J x \in \mathcal{H}_{J T J}(\bar{F})$. From (5) we obtain

$$
J x=J(\lambda I-T) f(\lambda)=\left(\bar{\lambda} J-J T J^{2}\right) f(\lambda)=\left(\bar{\lambda} I-T^{J}\right) J f(\lambda)
$$

The function $g(\bar{\lambda}):=J f(\lambda)$, is clearly defined for $\bar{\lambda} \notin \bar{F}$. Moreover, $g(\bar{\lambda})$ is analytic on $\mathbb{C} \backslash \bar{F}$. Therefore

$$
\left(\bar{\lambda} I-T^{J}\right) g(\bar{\lambda})=J x=y, \quad \text { for all } \bar{\lambda} \notin \bar{F},
$$

so $y \in \mathcal{H}_{T^{J}}(\bar{F})$, and hence $x=J y \in \mathcal{H}_{T^{J}}(\bar{F})$. Consequently, $\mathcal{H}_{T}(F) \subseteq$ $J\left(\mathcal{H}_{T^{J}}(\bar{F})\right)$.

To show the reverse inclusion observe that, since $J T^{J} J=T$, from the previous argument applied to $T^{j}$ we then have $\mathcal{H}_{T^{J}}(\bar{F}) \subseteq J \mathcal{H}_{T^{J}}(F)$, and hence

$$
J \mathcal{H}_{T^{J}}(\bar{F}) \subseteq J^{2} \mathcal{H}_{T^{J}}(F)=\mathcal{H}_{T^{J}}(F),
$$

so the first identity in (4) is proved. The second equality in (4) is immediate from the first one.

For every $T \in L(H)$ let $\rho_{T}(x):=\mathbb{C} \backslash \sigma_{T}(x)$ be the local resolvent of $T$ at $x$.

Lemma 2.6. If $T \in L(H)$ and $J$ is a conjugation then $\sigma_{T^{J}}(J x)=\overline{\sigma_{T}(x)}$ and $\overline{\sigma_{T}(J x)}=\sigma_{T^{J}}(x)$ for all $x \in H$.

Proof. Let $\lambda_{0} \in \rho_{T}(x)$. Then there exists an analytic function $f: \mathbb{D}_{0} \rightarrow H$, defined on an open disc $\mathbb{D}_{0}$ centered at $\lambda_{0}$ such that $(\lambda I-T) f(\lambda)=x$ for all $\lambda \in \mathbb{D}_{0}$. Then $J(\lambda I-T) f(\lambda)=J x$, and for all $\lambda \in \mathbb{D}_{0}$ we then have $J x=J(\lambda I-T) f(\lambda)=(\bar{\lambda} J-J T) f(\lambda)=\left(\bar{\lambda} J-J T J^{2}\right) f(\lambda)=\left(\bar{\lambda} I-T^{J}\right) J f(\lambda)$. Since $g(\bar{\lambda}):=J f(\lambda)$ is analytic for all $\bar{\lambda} \in \overline{\mathbb{D}_{0}}$, it then follows that $\bar{\lambda}_{0} \in$ $\rho_{T^{J}}(J x)$, so $\sigma_{T^{J}}(J x) \subseteq \overline{\sigma_{T}(x)}$.

Conversely, if $\lambda_{0} \in \rho_{T^{J}}(J x)$ there exists an analytic function $f: \mathbb{D}_{0} \rightarrow H$ defined on an open disc $\mathbb{D}_{0}$ centered at $\lambda_{0}$ such that $\left(\lambda I-T^{J}\right) f(\lambda)=J x$ for all $\lambda \in \mathbb{D}_{0}$. Hence

$$
(\bar{\lambda} I-T) J f(\lambda)=\left(\lambda J-J^{2} T J\right) f(\lambda)=J\left(\bar{\lambda}-T^{J}\right) f(\lambda)=J^{2} x=x,
$$

for all $\bar{\lambda} \in \overline{\mathbb{D}_{0}}$, so $\bar{\lambda} \in \rho_{T}(x)$, and hence $\overline{\sigma_{T}(x)} \subseteq \sigma_{T^{J}}(J x)$. The second equality may be proved in a similar fashion.

The quasi-nilpotent part of an operator $T \in L(X)$ is defined as

$$
H_{0}(T):=\left\{x \in X: \limsup _{n \rightarrow \infty}\left\|T^{n} x\right\|^{\frac{1}{n}}=0\right\},
$$

while the analytic core $K(T)$ is the set of all $x \in X$ such that there exists a sequence $\left(u_{n}\right)$ in $X$ and a constant $\delta>0$ such that $x=u_{0}, T u_{n+1}=u_{n}$ and $\left\|u_{n}\right\| \leq \delta^{n}\|x\|$ for every $n=0,1, \ldots$ Observe that $H_{0}(\lambda I-T)=\mathcal{X}_{T}(\{\lambda\}$, and $K(T)=\left\{x: 0 \in \rho_{T}(x)\right\}$ see [2, Chapter 2]. 
Theorem 2.7. Let $T \in L(H)$ and suppose that $J$ is a conjugation. Then

(i) $H_{0}(T)=J H_{0}\left(T^{J}\right)$ and $H_{0}\left(T^{J}\right)=J H_{0}(T)$.

(ii) $K(T)=J K\left(T^{J}\right)$ and $K\left(T^{J}\right)=J K(T)$.

(iii) $J \operatorname{ker}(\lambda I-T)^{n}=\operatorname{ker}\left(\bar{\lambda} I-T^{J}\right)^{n}$ for every $n \in \mathbb{N}$ and $\lambda \in \mathbb{C}$.

Proof. The equalities (i) are a consequence of Theorem 2.5, since $H_{0}(T)=$ $\mathcal{H}_{T}(\{0\})$ and $\mathcal{H}_{0}\left(T^{J}\right)=\mathcal{H}_{T^{J}}(\{0\})$.

(ii) To prove the equality $K(T)=J K\left(T^{J}\right)$, observe first that $K(T)=$ $H_{T}(\mathbb{C} \backslash\{0\})$, by $[2$, Theorem 2.20]. Let $x \in K(T)$. Then the local spectrum $\sigma_{T}(x) \subseteq \mathbb{C} \backslash\{0\}$. Since $\sigma_{T^{J}}(J x)=\overline{\sigma_{T}(x)}$ then $\sigma_{T^{J}}(J x) \subset \mathbb{C} \backslash\{0\}$. Hence $J x \in K\left(T^{J}\right)=H_{T^{J}}(\mathbb{C} \backslash\{0\})$. Conversely, if $y \in K\left(T^{J}\right)$ then $\sigma_{T^{J}}(y) \subseteq \mathbb{C} \backslash\{0\}$ implies that $\overline{\sigma_{T^{J}}(y)} \subseteq \mathbb{C} \backslash\{0\}$, from which $\sigma_{T}(y) \subseteq \mathbb{C} \backslash\{0\}$. Hence Jy $\in K(T)$, so $y=J^{2} y=J(J y) \in J K(T)$. Thus $K(T)=J K\left(T^{J}\right)$. The equality $K\left(T^{J}\right)=J K(T)$ may be obtained from the first part, since $T=J T^{J} J$.

(iii) Since $J^{2}=I$ it easily seen, via the classical Newton's binomial formula, that

$$
J(\lambda I-T)^{n}=\left(\bar{\lambda} I-T^{J}\right)^{n} J \text { for each } n \in \mathbb{N},
$$

and analogously

$$
J\left(\bar{\lambda} I-T^{J}\right)^{n}=(\lambda I-T)^{n} J \text { for each } n \in \mathbb{N} .
$$

To show (iii), let $x \in \operatorname{ker}(\lambda I-T)^{n}$. Then

$$
\left(\bar{\lambda} I-T^{J}\right)^{n} J x=J(\lambda I-T)^{n} x=0,
$$

thus $J x \in \operatorname{ker}\left(\bar{\lambda} I-T^{J}\right)^{n}$, i.e., $J \operatorname{ker}(\lambda I-T)^{n} \subseteq \operatorname{ker}\left(\bar{\lambda} I-T^{J}\right)^{n}$. To show the reverse inclusion, let $x \in \operatorname{ker}\left(\bar{\lambda} I-T^{J}\right)^{n}$. Then $\left(\bar{\lambda} I-T^{J}\right)^{n} x=0$ from which we obtain

$$
(\lambda I-T)^{n} J x=J\left(\bar{\lambda} I-T^{J}\right)^{n} x=0,
$$

so $J x \in \operatorname{ker}(\lambda I-T)^{n}$, hence $x=J(J x) \in J \operatorname{ker}(\lambda I-T)^{n}$.

The ascent of $T \in L(X)$ is the smallest positive integer $p=p(T)$, whenever it exists, such that ker $T^{p}=\operatorname{ker} T^{p+1}$. If such $p$ does not exist we let $p=+\infty$. Analogously, the descent of $T$ is defined to be the smallest integer $q=q(T)$, whenever it exists , such that $T^{q+1}(H)=T^{q}(H)$. If such $q$ does not exist we set $q=+\infty$. Note that if $p(T)$ and $q(T)$ are both finite then $p(T)=q(T)$. Moreover $\lambda$ is a pole of the resolvent if and only if $0<$ $p(\lambda I-T)=q(\lambda I-T)<\infty$.

Corollary 2.8. Let $T \in L(H)$ and suppose that $J$ is a conjugation. Then

(i) $p(\lambda I-T)<\infty \Leftrightarrow p\left(\bar{\lambda} I-T^{J}\right)<\infty$.

(ii) $q(\lambda I-T)<\infty \Leftrightarrow q\left(\bar{\lambda} I-T^{J}\right)<\infty$.

Proof. (i) Suppose that $\operatorname{ker}(\lambda I-T)^{n}=\operatorname{ker}(\lambda I-T)^{n+1}$. Then, from (iii) of Theorem 2.7, we have

$$
\operatorname{ker}\left(\bar{I}-T^{J}\right)^{n}=J \operatorname{ker}(\lambda I-T)^{n}=J \operatorname{ker}(\lambda I-T)^{n+1}=\operatorname{ker}\left(\bar{\lambda} I-T^{J}\right)^{n+1} .
$$


The converse follows in a similar way.

(ii) Assume that $(\lambda I-T)^{n}(H)=(\lambda I-T)^{n+1}(H)$ and let $y \in\left(\bar{\lambda} I-T^{J}\right)^{n}(H)$. Then $y=\left(\bar{\lambda} I-T^{J}\right)^{n}(x)$ for some $x \in H$, so by (6) we have

$J y=J\left(\bar{\lambda} I-T^{J}\right)^{n}(x)=(\lambda I-T)^{n} J x \subseteq(\lambda I-T)^{n}(H)=(\lambda I-T)^{n+1}(H)$.

Hence, there exists $z \in H$ such that $J y=(\lambda I-T)^{n+1}(z)$, from which we obtain

$$
y=J(J y)=J(\lambda I-T)^{n+1}(z)=\left(\bar{\lambda} I-T^{J}\right)^{n+1}(J z) \in\left(\bar{\lambda} I-T^{J}\right)^{n+1}(H),
$$

and, consequently, $\left(\bar{\lambda} I-T^{J}\right)^{n}(H) \subseteq\left(\bar{\lambda} I-T^{J}\right)^{n+1}(H)$. The converse inclusion is true for every operator, so the equality holds. The opposite implication follows by using a similar method.

Let iso $F$ denote the set of all isolated points of $F \subseteq \mathbb{C}$. An operator $T \in$ $L(X)$ is said to be polaroid, (respectively, a-polaroid), if every $\lambda \in$ iso $\sigma(T)$ (respectively, every $\lambda \in$ iso $\sigma_{\text {ap }}(T)$ ) is a pole of the resolvent. If $T$ is complex symmetric then, since $\sigma\left(T^{J}\right)=\overline{\sigma(T)}=\overline{\sigma_{\text {ap }}(T)}$, see [16, Lemma 4.1], we have

$$
T \text { is polaroid } \Leftrightarrow T \text { is } a-\text { polaroid } .
$$

Theorem 2.9. Let $T \in L(H)$ and let $J$ be a conjugation. Then $T$ is polaroid if and only if $T^{J}$ is polaroid.

Proof. Suppose that $T$ is polaroid and let $\lambda \in$ iso $\sigma\left(T^{J}\right)$. Then $\bar{\lambda} \in$ iso $\sigma(T)$, so $\bar{\lambda}$ is a pole of the resolvent of $T$ and hence $0<p(\bar{\lambda} I-T)=q(\bar{\lambda} I-T)<\infty$. From Corollary 2.8 it then follows that $0<p\left(\lambda I-T^{J}\right)=q\left(\lambda I-T^{J}\right)<\infty$, so $\lambda$ is a pole of the resolvent of $T^{J}$. Conversely, if $T^{J}$ is polaroid then $T=J T^{J} T$ is polaroid, by the first part of the proof.

Definition 2.10. $T \in L(X)$ has property $(Q)$ if $H_{0}(\lambda I-T)$ is closed for all $\lambda \in \mathbb{C}$, while $T \in L(X)$ is said to have property $H(p)$ if there exist a natural $p:=p(\lambda)$ such that:

$$
H_{0}(\lambda I-T)=\operatorname{ker}(\lambda I-T)^{p} \quad \text { for all } \lambda \in \mathbb{C} .
$$

The class of $H(p)$-operators is very large, it includes every generalized scalar operator, hyponormal operators, several generalization of hyponormal operators, and totally paranormal operators on Banach spaces, see $[2, \S 4.3]$. Every $H(p)$-operator is polaroid, see [2, Chapter 4].

Corollary 2.11. Let $T \in L(H)$ and $J$ a conjugation. Then $T$ has property $H(p)$ if and only if $T^{J}$ has property $H(p)$. In particular, if $T$ is complex symmetric then $T$ has property $H(p)$ if and only if $T^{*}$ does.

Proof. Suppose that $T$ has property $H(p)$. Then $H_{0}(\lambda I-T)=\operatorname{ker}(\lambda I-T)^{p}$, so $J H_{0}(\lambda I-T)=J \operatorname{ker}(\lambda I-T)^{p}$. By Theorem 2.7 it then follows that $H_{0}\left(\bar{\lambda} I-T^{J}\right)=\operatorname{ker}\left(\bar{\lambda} I-T^{J}\right)^{p}$, i.e., $T^{J}$ has property $H(p)$. Conversely, if $T^{j}$ has property $H(p)$ then $T=J T^{J} J$ has property $H(p)$. The second assertion is clear. 
Recall that $T \in L(X)$ on a Banach space $X$ has Dunford property $(C)$ if the analytic spectral subspace $X_{T}(F)$ is closed for all closed $F \subseteq C$. Note that if the quasi-nilpotent part $H_{0}(\lambda I-T)$ is closed then $T$ has SVEP at $\lambda$, by $[2$, Theorem 2.79$]$, so

$$
\text { property }(C) \Rightarrow \text { property }(Q) \Rightarrow \text { SVEP, }
$$

and examples of operators which have property $(Q)$ but not property $(C)$ may be found among convolution operators on group algebras, see [2, Chapter 2] and [20, Chapter 4]. Since property $(C)$ entails SVEP, if $T$ has property $(C)$ then $X_{T}(F)=\mathcal{X}_{T}(F)$ for every closed subset $F \subseteq \mathbb{C}$, in particular $H_{0}(\lambda I-T)=X_{T}(\{\lambda\})=\mathcal{X}_{T}(\{\lambda\})$. Evidently, from Theorem 2.7, for every conjugation $J$ we have

$$
T \text { has property }(Q) \Leftrightarrow T^{J} \text { has property }(Q) \text {. }
$$

Furthermore, fromTheorem 2.5 we also have that

$$
T \text { has property }(C) \Leftrightarrow T^{J} \text { has property }(C) \text {. }
$$

In the sequel for every subset $V \subseteq \mathbb{C}$ we shall denote by $V^{c l}$ the closure of $V$.

Recall that $T \in L(X)$ has property $(\delta)$ if $X=\mathcal{X}_{T}\left(V^{c l}\right)+\mathcal{X}_{T}\left(W^{c l}\right)$ for every open cover $\{V, W\}$ of $\mathbb{C}$. An operator $T \in L(X)$ is said to have Bishop's property $(\beta)$ if for every open subset $G \subseteq \mathbb{C}$ and every sequence $f_{n}: G \rightarrow H$ such that $(\lambda I-T) f_{n}(\lambda)$ converges uniformly to 0 in norm on compact subsets of $G$, then $f_{n}$ converges uniformly to 0 in norm on compact subsets of $G$.

Property $(\delta)$ and property $(\beta)$, are dual to each other, see [20], in the sense that $T$ has property $(\beta)$ (respectively, property $(\delta)$ ) if and only if $T^{\prime}$ has property $(\delta)$ (respectively, property $(\beta)$ ). Recall that $T \in L(X)$ is decomposable if has both properties $(\delta)$ and $\beta$ ).

For a complex symmetric operator $T$ we have, see [15, Theorem 2.1],

$T$ has property $(\delta) \Leftrightarrow T$ has property $(\beta) \Leftrightarrow T$ is decomposable.

Remark 2.12. The proof of [15, Theorem 2.1] uses the fact that $T^{*}$ has property $(\beta)$ if and only if $T$ has property $(\delta)$. There is a certain inaccuracy in [15], concerning some local spectral properties of the adjoint $T^{*}$. Indeed, many local spectral properties claimed in [15] have been established for the dual $T^{\prime}$ of a Banach space operator, see for instance [20, Theorem 2.5.5], and not for the adjoint $T^{*}$. Anyway these claims are correct, and in order to show that we recall the relationship between $T^{*}$ and the dual $T^{\prime}$. By the Frechét-Riesz representation theorem there exists a conjugated-linear isometry $U: H \rightarrow H^{\prime}$, $H^{\prime}$ the dual of $H$, that associates to every $y \in H$ the linear form defined $f_{y}(x):=\langle x, y\rangle$. Moreover, the dual and the adjoint of $T$ satisfy the equality

$$
\left(\bar{\lambda} I-T^{*}\right)=U^{-1}\left(\lambda I-T^{\prime}\right) U \text { for every } \lambda \in \mathbb{C} .
$$

Hence

$$
U\left(\bar{\lambda} I-T^{*}\right)=\left(\lambda I-T^{\prime}\right) U \quad \text { and } \quad\left(\bar{\lambda} I-T^{*}\right) U^{-1}=U^{-1}\left(\lambda I-T^{\prime}\right) .
$$

Let $x \in \mathcal{H}_{T^{*}}(F)$, for some closed $F \subseteq \mathbb{C}$. Then there exists an analytic function $f: \mathbb{C} \rightarrow H$ such that $\left(\lambda I-T^{*}\right) f(\lambda)=x$ for all $\lambda \in \mathbb{C} \backslash F$. From (8) 
we know that $U\left(\lambda I-T^{*}\right)=\left(\bar{\lambda} I-T^{\prime}\right) U$, so

$$
U x=U\left(\lambda I-T^{*}\right) f(\lambda)=\left(\bar{\lambda} I-T^{\prime}\right) U f(\lambda) \text { for all } \lambda \in \mathbb{C} \backslash F .
$$

The function $g(\bar{\lambda}):=U f(\lambda)$ for $\bar{\lambda} \in \mathbb{C} \backslash \bar{F}$ is analytic, so $U x \in \mathcal{H}_{T^{\prime}}(\bar{F})$. This shows that $U \mathcal{H}_{T^{*}}(F) \subseteq U \mathcal{H}_{T^{\prime}}(\bar{F})$. Analogously, it can be shown that $\mathcal{H}_{T^{\prime}}(\bar{F}) \subseteq \mathcal{H}_{T^{\prime}}(\bar{F})$ for every closed set $F \subseteq \mathbb{C}$, so $\mathcal{H}_{T^{\prime}}(\bar{F})=\mathcal{H}_{T^{\prime}}(\bar{F})$.

Now, if $T^{*}$ has property $(\delta)$ then $H=\mathcal{H}_{T^{*}}\left(V^{c l}\right)+\mathcal{H}_{T^{*}}\left(W^{c l}\right)$ for every cover $\{V, W\}$ of $\mathbb{C}$, so

$$
H=U H=U \mathcal{H}_{T^{*}}\left(V^{c l}\right)+U \mathcal{H}_{T^{*}}\left(W^{c l}\right)=\mathcal{H}_{T^{\prime}}\left(\overline{V^{c l}}\right)+\mathcal{H}_{T^{\prime}}\left(\bar{W}^{c l}\right),
$$

and hence, $T^{\prime}$ has property $(\delta)$. An analogous argument shows that if $T^{\prime}$ has property $(\delta)$ then $T^{*}$ has property $(\delta)$, so we have.

$$
T^{\prime} \text { has property }(\delta) \Leftrightarrow T^{*} \text { has property }(\delta) \text {, }
$$

By duality, $T^{\prime}$ has property $(\beta) \Leftrightarrow T=\left(T^{*}\right)^{*}$ has property $(\delta)$, and hence, by $(9)$, if and only if $\left(T^{*}\right)^{\prime}$ has property $(\delta)$, from which we conclude that

$$
T^{\prime} \text { has property }(\beta) \Leftrightarrow T^{*} \text { has property }(\beta) \text {. }
$$

Consequently,

$T^{\prime}$ is decomposable $\Leftrightarrow T^{*}$ is decomposable.

Theorem 2.13. Let $T \in L(H)$ and $J$ a conjugation on $H$. Then $T$ has property $(\delta)$ (respectively, property $(\beta)$, decomposable) if and only if $T^{J}$ has property $(\delta)$ (respectively, property $(\beta)$, decomposable). In particular, a complex symmetric operator $T$ has property $(\delta)$ (respectively, property $(\beta)$, decomposable) if and only if $T^{*}$ does.

Proof. Suppose that $T$ has property $(\delta)$ and let $\{V, W\}$ be a cover of $\mathbb{C}$. Then $\{\bar{V}, \bar{W}\}$ is an open cover of $\mathbb{C}$, and by Theorem 2.5 ,

$$
H=J H=J \mathcal{H}_{T}\left(\bar{V}^{c l}\right)+J \mathcal{H}_{T}\left(\bar{W}^{c l}\right)=\mathcal{H}_{T^{J}}\left(V^{c l}\right)+\mathcal{H}_{T^{J}}\left(W^{c l}\right),
$$

so $T^{J}$ has property $(\delta)$. In the same way, property $(\delta)$ for $T^{J}$ entails that $J T^{J} J=T$ has property $(\delta)$.

Suppose that $T$ has property $(\beta)$. Then $T^{\prime}$ and hence $T^{*}$ has property $(\delta)$, so $T^{* J}=\left(T^{J}\right)^{*}$ has property $(\delta)$, by the first part of the proof. Consequently, $T^{J}$ has property $(\beta)$. The reserve is clear, if $T^{J}$ has property $(\beta)$ then $T=J T^{J} J$ has property $(\beta)$.

The equivalence of decomposability for $T$ and $T^{J}$ is clear, since the decomposability is the union of the properties $(\beta)$ and $(\delta)$.

The result concerning property $(\beta)$ in Theorem 2.13 has been observed in [17], see the claim II in the proof of Theorem 3.7. The equivalence of property $(\beta)$ for symmetric operators for $T$ and $T^{*}$ has been established in [15], see the proof of Theorem 2.1. Our proof is more simple.

Lemma 2.14. Let $T \in L(X), X$ a Banach space, and $\lambda \in \rho(T)$. Then $\lambda(\lambda I-$ $T)^{-1} x \rightarrow x$ for every $x \in X$ as $|\lambda| \rightarrow+\infty$. 
Proof. Fix $x \in X$ and define $f(\lambda):=(\lambda I-T)^{-1} x: \rho(T) \rightarrow X$. It is known that $f(\lambda) \rightarrow 0$ when $|\lambda| \rightarrow+\infty$. We have $\lambda(\lambda I-T)^{-1} x-x=\lambda(\lambda I-T)^{-1} x-(\lambda I-T)(\lambda I-T)^{-1} x=T(\lambda I-T)^{-1} x \quad$ for $\lambda \in \rho(T)$, hence $\lambda(\lambda I-T)^{-1} x-x \rightarrow 0$, so $\lambda(\lambda I-T)^{-1} x \rightarrow x$ as $|\lambda| \rightarrow+\infty$.

Theorem 2.15. Let $T \in L(H)$, then $H_{0}\left(T^{*}\right) \subseteq K(T)^{\perp}$.

Proof. Let $x \in H_{0}\left(T^{*}\right)=\mathcal{H}_{T^{*}}(\{0\})$ and fix an arbitrary $y \in \mathcal{K}(T)$. We have to show that $\langle x, y\rangle=0$. As already observed $K(T)=\left\{x: 0 \in \rho_{T}(x)\right\}$, so there exist two analytic functions $f: \mathbb{C} \backslash\{0\} \rightarrow H$ and $g: \mathbb{D}_{0} \rightarrow H, \mathbb{D}_{0}$ an open disc centered at 0 , such that

$$
\left(\bar{\lambda} I-T^{\star}\right) f(\bar{\lambda})=x, \lambda \in \mathbb{C} \backslash\{0\}, \quad \text { and } \quad(\lambda I-T) g(\lambda)=y, \quad \lambda \in \mathbb{D}_{0} .
$$

Both the functions $f(\lambda)$ and $g\left(\lambda\right.$ are defined in $\mathbb{D}_{0} \backslash\{0\}$ and for $\mu \in \mathbb{D}_{0} \backslash\{0\}$ we have

$$
\langle f(\bar{\mu}), y\rangle=\langle f(\bar{\mu}),(\mu I-T) g(\mu)\rangle=\left\langle\left(\bar{\mu} I-T^{*}\right) f(\bar{\mu}), g(\mu)\right\rangle=\langle x, g(\mu)\rangle .
$$

Define

$$
h(\mu):=\left\{\begin{array}{l}
\langle f(\bar{\mu}), y\rangle \text { if } \mu \neq \mathbb{D}_{0}, \\
\langle x, g(\mu)\rangle \text { if } \mu \in \mathbb{D}_{0},
\end{array}\right.
$$

The function $h(\mu)$ is well-defined and is analytic on $\mathbb{C}$. Since $f(\bar{\mu})=(\bar{\mu} I-$ $\left.T^{*}\right)^{-1} x$ for all $\bar{\mu} \in \rho\left(T^{*}\right)$, see $[2$, Remark 2.11] and $f(\bar{\mu}) \rightarrow 0$ for $|\bar{\mu}| \rightarrow+\infty$, then $h(\mu) \rightarrow 0$ as $|\mu| \rightarrow+\infty$, so, by the classical Liouville theorem, $h \equiv 0$ on C. From Lemma 2.14 we have also have $\bar{\mu}\left(\bar{\mu} I-T^{*}\right)^{-1} x=-x$, as $|\mu| \rightarrow+\infty$, $\bar{\mu} \in \rho\left(T^{*}\right)$, hence

$$
\langle x, y\rangle=\lim _{|\mu| \rightarrow+\infty}\left\langle\bar{\mu}\left(\bar{\mu} I-T^{*}\right)^{-1} x, y\right\rangle=\lim _{|\mu| \rightarrow+\infty}\langle\bar{\mu} f(\bar{\mu}), y\rangle=\mu h(\mu)=0,
$$

so $x \in K(T)^{\perp}$, as desired.

Next we want to show that if $T$ is decomposable then $H_{0}\left(T^{*}\right)=K(T)^{\perp}$. To do this we need some preliminary results. Suppose that $M$ is a closed $T$ invariant subspace of a Banach space $X$ and denote by $T / M: X / M \rightarrow X / M$ the canonical quotient mapping defined on the quotient $X / M$ by $(T / M)(x+$ $M):=T x+M$.

For an open disc $\mathbb{D}$ of $\mathbb{C}$ centered at 0 , let $\mathbf{D}$ denote its closure.

Lemma 2.16. Suppose that $T \in L(X), X$ a Banach space, is decomposable. If $M:=X_{T}(\mathbb{C} \backslash \mathbb{D})$ then $\sigma(T / M)$ is contained in $\mathbf{D}$.

Proof. This follows as a particular case of Theorem 1.2.23, part (b), of [20], by taking $F=\mathbb{C} \backslash \mathbb{D}$.

If $Y$ is a closed $T$-invariant subspace by $T \mid Y$ we denote the restriction of $T$ to $Y$.

Lemma 2.17. Suppose that $T \in L(H)$ is decomposable. If $\mathbb{D}$ is an open disc centered at 0 then $H_{T}(\mathbb{C} \backslash \mathbb{D})^{\perp} \subseteq H_{T^{*}}(\mathbf{D})$. 
Proof. Let $M:=X_{T}(\mathbb{C} \backslash \mathbb{D})$. Recall that $M$ is a closed invariant subspace of $T$, since a decomposable operator has property $(C)$, while $M^{\perp}$ is a closed subspace invariant under $T^{*}$. We show first that

$$
\sigma\left(T^{*} \mid M\right) \subseteq \mathbf{D}
$$

If $S: M^{\perp} \rightarrow H / M$ is defined by $S(x)=x+M$ for every $x \in M^{\perp}$, then $S$ is bijective and an isometry. It is easily seen that $S\left(T^{*} \mid M^{\perp}\right)=(T / M)^{*} S$, so $T^{*} \mid M^{\perp}$ and $(T / M)^{*}$ are similar. Therefore,

$$
\sigma\left(T^{*} \mid M^{\perp}\right)=\sigma(T / M)^{*}=\overline{\sigma(T / M)} .
$$

By Lemma 2.16 then

$$
\sigma\left(T^{*} \mid M^{\perp}\right)=\sigma\left(T^{*} \mid X_{T}(\mathbb{C} \backslash \mathbb{D})^{\perp}\right) \subseteq \mathbf{D},
$$

since $\mathbf{D}$ is the closure of $\overline{\mathbb{D}}=\mathbb{D}$. Thus, the inclusion (10) is proved. From part (e) of $\left[20, \quad\right.$ Proposition 1.2.16] we then obtain $H_{T}(\mathbb{C} \backslash \mathbb{D})^{\perp}$ $=M^{\perp} \subseteq H_{T^{*}}(\mathbf{D})$.

Theorem 2.18. Let $T \in L(H)$ be decomposable then $H_{0}\left(T^{*}\right)=K(T)^{\perp}$ and $H_{0}(T)=K\left(T^{*}\right)^{\perp}$.

Proof. To show the equality $H_{0}\left(T^{*}\right)=K(T)^{\perp}$, let $\left\{\mathbf{D}_{\alpha}\right\}_{\alpha}$ denote the set of all closed discs of $\mathbb{C}$ centered at 0 . Since $T$ has SVEP we have

$$
H_{0}\left(T^{*}\right)=\mathcal{H}_{T^{*}}(\{0\})=H_{T^{*}}(\{0\})=\bigcap_{\alpha} H_{T^{*}}\left(\mathbf{D}_{\alpha}\right),
$$

see $\left[2\right.$, Theorem 2.13, part (iv)]. To show the equality $H_{0}\left(T^{*}\right)=K(T)^{\perp}$ we need to prove, by Theorem 2.15, the inclusion $K(T)^{\perp} \subseteq H_{0}\left(T^{*}\right)$, and for this it suffices to prove that $K(T)^{\perp} \subseteq H_{T^{*}}(\mathbf{D})$, where $\mathbf{D}$ is any closed disc centered at 0 . Evidently,

$$
H_{T}(\mathbb{C} \backslash \mathbb{D}) \subseteq H_{T}(\mathbb{C} \backslash\{0\})=K(T),
$$

so $K(T)^{\perp} \subseteq H_{T}(\mathbb{C} \backslash \mathbf{D})^{\perp}$ and $H_{T}(\mathbb{C} \backslash \mathbb{D})^{\perp} \subseteq H_{T^{*}}(\mathbf{D})$, by Lemma 2.16 , so the proof of the first equality is complete. The second equality is clear, since $T^{\prime}$, and hence $T^{*}$, is decomposable, by Remark 2.12, we have

$$
H_{0}(T)=H_{0}\left(\left(T^{*}\right)^{*}\right)=K\left(T^{*}\right)^{\perp} .
$$

Remark 2.19. It should be noted that the identity $K(T)=H_{0}\left(T^{*}\right)^{\perp}$ in general does not hold even if $T$ is decomposable. For instance, if $T \in L(H)$ is Riesz operator which has infinite spectrum then $T$ is decomposable, but $K(T)$ is not closed, since in this case $\sigma(T)$ would be finite, see [21]. Hence $K(T) \neq H_{0}\left(T^{*}\right)^{\perp}$, since $H_{0}\left(T^{*}\right)^{\perp}$ is closed.

Corollary 2.20. Suppose that $T \in L(H)$ has property $(\delta)$. If $T$ is $J$-complex symmetric, then $H_{0}(T)=J K(T)^{\perp}$. If $K(T)$ is closed then $K(T)=J H_{0}(T)^{\perp}$.

Proof. Observe first that for every subset $A \subseteq H$ we have $J A^{\perp}=(J A)^{\perp}$. Indeed, if $z \in J A^{\perp}$ then $z=J x$ where $\langle x, a\rangle=0$ for every $a \in A$. Thus $J z=x$ and hence

$$
\langle z, J a\rangle=\left\langle J^{2} z, J a\right\rangle=\langle a, J z\rangle=\langle a, x\rangle=\overline{\langle x, a\rangle}=0
$$


so $z \in(J A)^{\perp}$. Conversely, if $y \in(J A)^{\perp}$ then $\langle y, J a\rangle=0$ for every $a \in A$, so

$$
0=\langle y, J a\rangle=\left\langle J^{2} y, J a\right\rangle=\langle a, J y\rangle,
$$

hence $\langle J y, a\rangle=\overline{\langle a, J y\rangle}=0$. Thus, $J y \in A^{\perp}$ and hence $J^{2} y=y \in J A^{\perp}$.

Now, $T$ is decomposable, by Theorem 2.13, so, by Theorem $2.18, H_{0}(T)=$ $K\left(T^{*}\right)^{\perp}$. By Theorem 2.7 we have $K\left(T^{*}\right)=J K(T)$, hence $H_{0}(T)=$ $[J K(T)]^{\perp}=J K(T)^{\perp}$. The second equality is clear: $K(T)=H_{0}\left(T^{*}\right)^{\perp}=$ $\left[J H_{0}(T)\right]^{\perp}=J H_{0}(T)^{\perp}$.

It is known that for every operator on a Banach space $0 \in$ iso $\sigma(T)$ if and only if both $H_{0}(T)$ and $K(T)$ are closed and $H=K(T) \oplus H_{0}(T)$, see [2, Theorem 2.46]. Evidently, if $T$ is decomposable then $T$ has property $(Q)$, so $H_{0}(T)$ is closed. From Corollary 2.20 we readily obtain:

Corollary 2.21. Suppose that $T \in L(H)$ is $J$-complex symmetric and has property $(\delta)$. Then $0 \in$ iso $\sigma(T)$ if and only if $K(T)$ is closed and $H=$ $K(T) \oplus J K(T)^{\perp}$, or if and only if $K(T)$ is closed and $H=H_{0}(T) \oplus J H_{0}(T)^{\perp}$.

\section{Weyl Type Theorems for Operator Matrices}

In this section we give some results concerning Weyl type theorems for complex symmetric operators. According [8] an operator $T \in L(X)$ is said to be hereditarily polaroid if any restriction on a closed invariant subspace is polaroid. Since the restriction $T \mid M$ on a closed invariant subspace $M$ of an operator $T$ which has property $H(p)$ has also property $H(p)$, it then follows that every $H(p)$-operator is hereditarily polaroid.

Let $\mathcal{H}(\sigma(T))$ denote the set of all analytic functions defined on an open disc which contains the spectrum and let $f(T)$ be defined by means of the classical functional calculus.

Theorem 3.1. Le $T \in L(H)$ be complex symmetric. If $T$ has property $(\delta)$, then both $f(T)$ and $f\left(T^{*}\right)$ have property $H(p)$ for every $f \in \mathcal{H}(\sigma(T))$,

Proof. A remarkable result of [18] shows that if $T$ is $J$-complex symmetric and $T$ has property $(\delta)$ then there exists a scalar extension of $T$, hence $T$ is subscalar. By Theorem 4.40 of [2] it then follows that $T$ has the property $H(p)$. A result due to Oudghiri shows that if $T$ has the property $H(p)$, then $f(T)$ has property $H(p)$ for every $f \in \mathcal{H}(\sigma(T))$, see [22]. Since property $(\delta)$ from $T$ is transferred to $T^{*}=T^{J}$, the same argument above shows that $f\left(T^{*}\right)$ has property $H(p)$ for every $f \in \mathcal{H}(\sigma(T))$.

Denote by $\sigma_{\mathrm{w}}(T)$ and $\sigma_{\mathrm{uw}}(T)$ the Weyl and upper semi-Weyl spectra, respectively, (see [2] for definitions). The Weyl type theorems are defined as follows: an operator $T \in L(X)$ is said to verify Weyl's theorem if $\sigma(T) \backslash \sigma_{\mathrm{w}}(T)=$ $\pi_{00}(T)$, where $\pi_{00}(T):=\{\lambda \in$ iso $\sigma(T): 0<\alpha(\lambda I-T)<\infty\}$. The operator $T \in L(X)$ is said to verify a-Weyl's theorem if $\sigma_{\text {ap }}(T) \backslash \sigma_{\text {uw }}(T)=\pi_{00}^{a}(T)$, where $\pi_{00}^{a}(T):=\left\{\lambda \in\right.$ iso $\left.\sigma_{\text {ap }}(T): 0<\alpha(\lambda I-T)<\infty\right\}$, while $T \in L(X)$ is said to verify property $(\omega)$ if $\sigma_{\text {ap }}(T) \backslash \sigma_{\text {uw }}(T)=\pi_{00}(T)$. Either $a$-Weyl's 
theorem and property $(w)$ entails Weyl's theorem, see [2, Chapter 6]. In [15] it has been proved for a complex symmetric operator that

$T$ satisfies Weyl's theorem $\Leftrightarrow T^{*}$ does,

and in [17], see the Claim in the proof of Theorem 3.24, it is shown that all Weyl type theorems are equivalent for a complex symmetric operator $T$. The following result improves that of [16, Theorem 3.7].

Recall that an operator $T \in L(H)$ is said to be algebraic if there exists a polynomial $h$ such that $h(T)=0$. The SVEP is preserved under commuting algebraic perturbations, see [5], and is also preserved under the functional calculus, see [2, Chapter 2], i.e., if $T$ has SVEP then $f(T)$ has SVEP for every $f \in \mathcal{H}(\sigma(T))$. Moreover, if $T$ is polaroid then $f(T)$ is polaroid for every $f \in \mathcal{H}(\sigma(T))$, see $[1]$.

Theorem 3.2. Let $T \in L(H)$ be complex symmetric and suppose that $T$ has property $(\delta), K \in L(H)$ an algebraic operator which commutes with $T$. Then

(i) both $f(T+K)$ and $f\left(T^{*}+K^{*}\right)$ satisfy all Weyl type theorems for every $f \in \mathcal{H}(\sigma(T+K))$.

(ii) $f(T)+K$ and $f\left(T^{*}\right)+K^{*}$ satisfy all Weyl type theorems for every $f \in \mathcal{H}(\sigma(T))$.

In particular, $T+K$ and $T^{*}+K^{*}$ satisfy all Weyl type theorems.

Proof. (i) We already have observed that $T$ is subscalar and hence hereditarily polaroid. Since $T K=K T$ this implies, see [2, Theorem 4.32], that $T+K$ is polaroid, so $f(T+K)$ is polaroid. Furthermore since $T$ has SVEP, then $T+K$ has SVEP, and hence $f(T+K)$ has SVEP for every $f \in \mathcal{H}(\sigma(T+K))$. Finally, from [2, Theorem 6.13] we conclude that $f(T+K)$ satisfies all Weyl type theorems. Since $T$ is complex symmetric, the SVEP for $T$ entails the SVEP for $T^{*}$, and hence the SVEP for $f\left(T^{*}+K^{*}\right)$. Moreover, as above, $f\left(T^{*}+K^{*}\right)$ is polaroid, so, $f\left(T^{*}+K^{*}\right)$ satisfy all Weyl type theorems.

(ii) It follows by using similar arguments,

We now give some results concerning Weyl type theorems for operator matrices $\left(\begin{array}{ll}A & B \\ C & D\end{array}\right)$, complementary to those given in [15] and [17].

Lemma 3.3. Let $A, B, C, D \in L(H)$ and $\mathbf{T}:=\left(\begin{array}{cc}A & B \\ 0 & D\end{array}\right)$ or $\mathbf{S}:=\left(\begin{array}{cc}A & 0 \\ C & D\end{array}\right)$. If both $A$ and $D$ have property $(\beta)$ then $\mathbf{T}$ has property $(\beta)$. If both $A$ and $D$ have property $(\delta)$ then $\mathbf{S}$ has property $(\delta)$. In particular, this holds if $D=A^{J}$, for every conjugation $J$ on $H$, and $A$ has property $(\beta)$ (respectively, property $(\delta))$.

Proof. The assertion concerning property $(\beta)$ for $\mathbf{T}$ has been proved in [17], see Claim 1 in the proof of Theorem 3.7. If both $A$ and $D$ have property $(\delta)$, then $A^{*}$ and $D^{*}$ have property $(\beta)$, so, $\mathbf{S}^{*}=\left(\begin{array}{rr}A^{*} & C^{*} \\ 0 & D^{*}\end{array}\right)$ has property $(\beta)$, by the first assertion, and hence $\mathbf{S}$ has property $(\delta)$. The last assertion is clear, by Theorem 2.13 . 
Define, for $A, B \in L(H)$ and for a conjugation $J$ on $H$, the operator matrices

$$
\mathbf{T}_{1}:=\left(\begin{array}{cc}
A & B \\
0 & A^{J}
\end{array}\right), \quad \mathbf{T}_{2}:=\left(\begin{array}{cc}
A & B \\
0 & A
\end{array}\right), \quad \mathbf{T}_{3}:=\left(\begin{array}{cc}
A & B \\
0 & B
\end{array}\right), \quad \mathbf{T}_{4}:=\left(\begin{array}{cc}
A & B \\
0 & B^{J}
\end{array}\right) .
$$

Lemma 3.4. Suppose that $A$ and $B$ are complex symmetric. If $A$ has SVEP then $\mathbf{T}_{1}$ and $\mathbf{T}_{2}$ have SVEP. If both $A$ and $B$ have SVEP, then $\mathbf{T}_{3}$ and $\mathbf{T}_{4}$ have SVEP.

Proof. An upper triangular operator matrix has SVEP if all the operators on the main diagonal have SVEP.

In the sequel, for any conjugation $J$ on $H$ we set

$$
\mathbf{J}:=\left(\begin{array}{cc}
0 & J \\
J & 0
\end{array}\right) .
$$

Evidently, $\mathbf{J}$ is a conjugation on $H \oplus H$.

Theorem 3.5. Let $A, B \in L(H)$ be J-complex symmetric and let $\mathbf{T}_{i}, i=$ $1,2,3,4$ be the operator matrices defined above.

(i) If $A$ is decomposable then all Weyl type theorems hold for $f\left(\mathbf{T}_{i}\right)$ and $f\left(\mathbf{T}_{i}^{*}\right), i=1,2$, for every $f \in \mathcal{H}\left(\sigma\left(\mathbf{T}_{i}\right)\right)$.

(ii) If $A$ and $B$ are decomposable then all Weyl type theorems hold for $f\left(\mathbf{T}_{i}\right)$ and $f\left(\mathbf{T}_{i}\right)^{*}, i=3,4$, for every $f \in \mathcal{H}\left(\sigma\left(\mathbf{T}_{i}\right)\right)$.

(iii) If $A J=J A$ then $\mathbf{T}_{1}=\mathbf{T}_{2}$ and all Weyl type theorems hold for $f\left(\mathbf{T}_{1}\right)$ and $f\left(\mathbf{T}_{1}\right)^{*}$, for every $f \in \mathcal{H}\left(\sigma\left(\mathbf{T}_{1}\right)\right)$.

Proof. Observe first that all $\mathbf{T}_{i}$ are $\mathbf{J}$-complex symmetric. For instance, $A^{J}=$ $A^{*}$, so $\mathbf{T}_{1}^{*}=\left(\begin{array}{cc}A^{\star} & 0 \\ B^{*} & A^{*}\end{array}\right)$, and it is easy to check that $\mathbf{T}_{1} \mathbf{J}=\mathbf{J} \mathbf{T}_{1}^{*}$. Analogously, $\mathbf{T}_{2}, \mathbf{T}_{3}$ and $\mathbf{T}_{4}$ are $\mathbf{J}$-complex symmetric.

(i) If $A$ is decomposable then $A$ has property $(\beta)$, so, by Lemma $3.3, \mathbf{T}_{1}$ has property $(\beta)$, or equivalently has property $(\delta)$, hence Theorem 3.2 applies. The same reasoning may be used for $\mathbf{T}_{2}$, since $A^{J}=A^{*}$ is also decomposable, and hence, by Lemma $3.3, \mathbf{T}_{2}$ has property $(\beta)$.

(ii) If $A$ and $B$ are decomposable then $\mathbf{T}_{i}, i=1,2$ have property $(\beta)$, and hence we are in the same situation of part (i).

(iii) The condition $A J=J A$ entails, by (2), that $A$ is selfadjoint, so $A$ is decomposable.

We now consider the case where $D$ is the transpose $A^{* J}=J A^{*} J$. Set

$$
\mathbf{T}:=\left(\begin{array}{cc}
A & B \\
0 & J A^{*} J
\end{array}\right) \quad \text { and } \quad \mathbf{S}:=\left(\begin{array}{cc}
A & 0 \\
C & J A^{*} J
\end{array}\right) .
$$

Theorem 3.6. Let $A, B, C \in L(H)$,

(i) If $A$ is decomposable, and $B$ is $J$-complex symmetric, then all Weyl type theorem hold for $f(\mathbf{T})$ and $f\left(\mathbf{T}^{*}\right)$ for every $f \in \mathcal{H}(\sigma(\mathbf{T}))$.

(ii) If $A$ is decomposable, and $C$ is J-complex symmetric, then all Weyl type theorem hold for $f(\mathbf{S})$ and $f(\mathbf{S})^{*}$ for every $f \in \mathcal{H}(\sigma(\mathbf{S}))$. 
Proof. (i) The adjoint of $\mathbf{T}$ is the operator $\mathbf{T}^{*}:=\left(\begin{array}{cc}A^{*} & 0 \\ B^{*} & J A J\end{array}\right)$ and it is easily seen that $\mathbf{T} \mathbf{J}=\mathbf{J} \mathbf{T}^{*}$, so $\mathbf{T}$ is $\mathbf{J}$-complex symmetric. Since $A$ is decomposable then $A^{*}$ is decomposable, so, by Theorem 2.13, also $J A^{*} J$ is decomposable. In particular, $A$ and $J A^{*} J$ have property $(\beta)$. and hence, by Theorem $3.3, \mathbf{T}$ has property $(\beta)$. Being $\mathbf{T}$ complex symmetric then, by Theorem 2.13 , $\mathbf{T}$ has property $(\delta)$. By Theorem 3.2, we then conclude that Weyl type theorems hold for $f(\mathbf{T})$ and $f(\mathbf{T})^{*}$ for all $f \in \mathcal{H}(\sigma(\mathbf{T}))$.

(ii) We have $\mathbf{S}^{*}=\left(\begin{array}{cc}A^{*} & C^{*} \\ 0 & J A J\end{array}\right)$, and since $C$ is $J$-complex symmetric we easily have $\mathbf{S J}=\mathbf{J S}^{*}$, hence $\mathbf{S}$ is $\mathbf{J}$-complex symmetric. As above $J A^{*} J$ is decomposable and this implies that $J A J=\left(J A^{*} J\right)^{*}$ is decomposable. Since decomposability entails property $(\delta)$, by Lemma 3.3 then $\mathbf{S}$ has property $(\delta)$ and hence, by Theorem 3.2 , both $f(\mathbf{S})$ and $f\left(\mathbf{S}^{*}\right)$ satisfy all Weyl type theorems for all $f \in \mathcal{H}(\sigma(\mathbf{S}))$.

It should be noted that in Theorem 3.6 the assumption that $B$ or $C$ is $J$-complex symmetric is not needed in order to get the decomposability of $\mathbf{T}$, see also [17, Theorem 3.7].

Example 3.7. Let $H^{2}(\boldsymbol{\Gamma})$ denote the classical Hardy space, where $\boldsymbol{\Gamma}$ denote the unit circle, and $H^{\infty}(\boldsymbol{\Gamma})=L^{\infty}(\boldsymbol{\Gamma}) \cap H^{2}(\boldsymbol{\Gamma})$. For $\phi \in L^{\infty}(\boldsymbol{\Gamma})$, let $T_{\phi}$ be the Toeplitz operator with symbol $\phi$, defined by

$$
T_{\phi} f:=P(\phi f) \quad \text { for all } f \in H^{2}(\boldsymbol{\Gamma}),
$$

where $P$ is the orthogonal projection of $L^{2}(\boldsymbol{\Gamma})$ onto $H^{2}(\boldsymbol{\Gamma})$. Define $M_{\phi}$ : $L^{2}(\boldsymbol{\Gamma}) \rightarrow L^{2}(\boldsymbol{\Gamma})$ by $M_{\phi} f=\phi f \quad$ for all $f \in L^{2}(\boldsymbol{\Gamma})$, where $\phi f$ is the point-wise product. It is known that $\phi \in H^{\infty}(\boldsymbol{\Gamma})$, then $T_{\phi}$ is subnormal, since is the restriction of the normal operator $M_{\phi}$ to $H^{2}(\boldsymbol{\Gamma})$. Every subnormal operator is hyponormal, see Conway [6, Proposition 2.4.2], and hence $T_{\phi}$ has property $H(p)$, see [2, Chapter 4]. We also have that, for every $\phi \in H^{\infty}(\mathbf{T}), T_{\phi}$ has property $(\beta)$, see $\left[20\right.$, Theorem2.4.4]. In [3] it is shown that if $\phi \in H^{\infty}(\boldsymbol{\Gamma})$ then $f\left(T_{\phi}\right)^{*}$ satisfies Weyl type theorems for every $f \in \mathcal{H}\left(\sigma\left(T_{\phi}\right)\right)$.

In general, a Toeplitz operator is not complex symmetric and in [19] some conditions are given in order a Toeplitz operator to be $J$-complex symmetric with respect a suitable conjugation $J$. Let $T_{\psi}$, with $\psi \in L^{\infty}(\boldsymbol{\Gamma})$ be $J$-complex symmetric, and consider the matrix operator

$$
\mathbf{S}:=\left(\begin{array}{cc}
T_{\phi} & T_{\psi} \\
0 & J T_{\bar{\phi}} J
\end{array}\right) \text {, where } \phi \in H^{\infty}(\boldsymbol{\Gamma}) .
$$

As observed above the Toeplitz operator $T_{\bar{\phi}}$ has property $(\beta)$, and hence also $J T_{\bar{\phi}} J$ has property $(\beta)$. By Theorem 3.3 then $\mathbf{S}$ has property $(\beta)$. Since $T_{\bar{\phi}}=T_{\phi}^{*}$, the argument in the proof of Theorem 3.6, shows that $\mathbf{S}$ is $\mathbf{J}$ complex symmetric. Consequently, from the equivalences $(7)$, we deduce that $\mathbf{S}$ has property $(\delta)$ and hence has property $H(p)$. From that we conclude that $\mathbf{S}$ satisfies all Weyl type theorems.

Example 3.8. Let us consider on the classical Hardy space $H^{2}(\mathbb{D})$ the composition operator $T_{\gamma}:=f \circ \gamma$, where $\gamma: \mathbb{D} \rightarrow \mathbb{D}$ is an automorphism. The 
automorphism $\gamma$ is called parabolic if

$$
\gamma(z)=\frac{a z+\bar{b}}{b z+\bar{a}} \quad \text { for all } z \in \mathbb{D}
$$

where $a, b \in \mathbb{C}$ satisfy $|a|^{2}-|b|^{2}=1$ and $\operatorname{Im} a=|b|$, while $\gamma$ is said to be elliptic if $\operatorname{Im} a>|b|$. By [23] we know that parabolic or elliptic composition operators are generalized scalars, hence decomposable and have property $H(p)$. Define $(J f)(z) .=\overline{f(\bar{z})}$. Then $J$ is a conjugation on $H^{2}(\mathbb{D})$, and the only composition operators $J$-complex symmetric are normal operators, see Remark 3.4 of [17]. As observed in [17, Corollary 3.8], if $\gamma$ is either parabolic or elliptic and $\phi$ is an analytic selfmap of $\mathbb{D}$ then $\mathbf{T}:=\left(\begin{array}{cc}T_{\phi} & T_{\psi} \\ 0 & J T_{\gamma}{ }^{*} J\end{array}\right)$ is decomposable. Suppose now that $T_{\psi}$ is $J$-complex symmetric, i.e. $T_{\psi}$ is normal. By Theorem 3.6 then $\mathbf{T}$ is $\mathbf{J}$-complex symmetric and $f(\mathbf{T})$ satisfies all Weyl type theorems for every $f \in \mathcal{H}(\sigma(\mathbf{T}))$.

Theorem 3.9. Suppose that $\mathbf{T} \in L(H \oplus H)$ is the main diagonal matrix $\mathbf{T}:=\left(\begin{array}{ll}A & 0 \\ 0 & B\end{array}\right)=A \oplus B$. Then $\mathbf{T}$ has property $(C)$ if and only if both $A, B$ have property $(C)$. Analogous statements hold for property $(Q)$ property $(\delta)$, property $(\beta)$. Moreover, if $A, B$ are $H(p)$-operators then $\mathbf{T}$ is a $H(p)$-operator.

Proof. Set $X:=H \oplus H$ and $F$ be any closed subset of $\mathbb{C}$. If $x \in \mathcal{H}_{A}(F)$ and $y \in \mathcal{H}_{B}(F)$, then there exist two analytic functions $f_{1}, f_{2}: \mathbb{C} \backslash F \rightarrow H$ such that $(\lambda I-A) f_{1}(\lambda)=x$ and $(\lambda I-B) f_{2}(\lambda)=y$. It is immediate to see thay $(\lambda \mathbf{I}-\mathbf{T})\left(f_{1}(\lambda) \oplus f_{2}(\lambda)\right)=x \oplus y$, so $x \oplus y \in \mathcal{H}_{\mathbf{T}}(F)$. Therefore, $\mathcal{H}_{A}(F) \oplus \mathcal{H}_{B}(F) \subseteq \mathcal{X}_{\mathbf{T}}(F)$. The opposite inclusion may be proved by using a similar argument, hence

$$
\mathcal{X}_{\mathbf{T}}(F)=\mathcal{H}_{A}(F) \oplus \mathcal{H}_{B}(F) \text { for every closed subset of } \mathbb{C} \text {. }
$$

Suppose that $A, B$ have property $(C)$, so both $\mathcal{H}_{A}(F)$ and $\mathcal{H}_{B}(F)$ are closed. Consequently, $\mathcal{X}_{\mathbf{T}}(F)$ is closed, and hence $\mathbf{T}$ has property $(C)$. From the equality (11) we deduce also the converse.

By considering the case where $F:=\{\lambda\}$ we deduce that if both $A, B \in$ $L(H)$ have property $(Q)$ if and only if $\mathbf{T}$ has property $(Q)$.

Suppose that $A$ and $B$, have property $(\delta)$. Then $H=\mathcal{H}_{A}\left(V^{c l}\right)+$ $\mathcal{H}_{A}\left(W^{c l}\right)$ and $H=\mathcal{H}_{B}\left(V^{c l}\right)+\mathcal{H}_{B}\left(W^{c l}\right)$ for every open cover $\{V, W\}$ of C. From this we obtain

$$
\begin{aligned}
X & =H \oplus H=\left[\mathcal{H}_{A}\left(V^{c l}\right)+\mathcal{H}_{A}\left(W^{c l}\right)\right] \oplus \mathcal{H}_{B}\left(V^{c l}\right)+\mathcal{H}_{B}\left(W^{c l}\right) \\
& =\left[\mathcal{H}_{A}\left(V^{c l}\right) \oplus \mathcal{H}_{B}\left(V^{c l}\right)\right]+\left[\mathcal{H}_{A}\left(W^{c l}\right) \oplus \mathcal{H}_{B}\left(W^{c l}\right)\right] \\
& =\mathcal{H}_{\mathbf{T}}\left(V^{c l}\right)+\mathcal{H}_{\mathbf{T}}\left(V^{c l}\right),
\end{aligned}
$$

hence $\mathbf{T}$ has property $(\delta)$ and the converse is also true, i.e., property $(\delta)$ for T entails property $(\delta)$ for $A$ and $B$.

Suppose that $A$ and $B$ have property $(\beta)$. By duality then $A^{*}$ and $B^{*}$ have property $(\delta)$, so, $A^{*} \oplus B^{*}=(A \oplus B)^{*}$ has property $(\delta)$ and hence $A \oplus B$ has 
property $(\beta)$.

Finally suppose that $A$ and $B$ are $H(p)$-operators. If $\lambda \in \mathbb{C}$ then

$$
H_{0}(\lambda I-A \oplus B)=H_{0}(\lambda I-A) \oplus H_{0}(\lambda I-B)=\operatorname{ker}(\lambda I-A)^{p} \oplus \operatorname{ker}(\lambda I-B)^{q}
$$

for some $p, q \in \mathbb{N}$. If $\nu:=\max \{p, q\}$ then

$$
H_{0}(\lambda I-A \oplus B)=\operatorname{ker}(\lambda I-A)^{\nu} \oplus \operatorname{ker}(\lambda I-B)^{\nu}=\operatorname{ker}(\lambda I-A \oplus B)^{\nu},
$$

thus $\mathbf{T}=A \oplus B$ has property $H(p)$. It is easy to check that also the converse holds.

Corollary 3.10. Let $A, C \in L(H)$ and suppose that $C$ is $J$-complex symmetric. If $\mathbf{T}:=\left(\begin{array}{ll}A & 0 \\ C & A\end{array}\right)$, then $f(\mathbf{T})$ satisfies all Weyl type theorems for all $f \in \mathcal{H}(\sigma(\mathbf{T}))$.

Proof. We have $\mathbf{T}^{*}:=\left(\begin{array}{cc}A^{*} & C^{*} \\ 0 & A^{*}\end{array}\right)$ and $\mathbf{T J}=\mathbf{J} \mathbf{T}^{*}$ hence $\mathbf{T}$ is $\mathbf{J}$-complex symmetric. If $A$ is decomposable then $\mathbf{T}$ is decomposable, and in particular has property $(\delta)$, so Theorem 3.2 applies.

The case of the operator matrices having form $\mathbf{T}:=\left(\begin{array}{cc}0 & A \\ B & 0\end{array}\right)$ is little more complicated. Recall first that if $X$ is a Banach space and $f: \mathcal{U} \rightarrow \mathbb{C}$ an analytic function defined on a open set $\mathcal{U} \subseteq \mathbb{C}$ containing the spectrum of $T$. Then

$$
\mathcal{X}_{f(T)}(F)=\mathcal{X}_{T}\left(f^{-1}(F)\right) \text { for all closed sets } F \subseteq \mathbb{C},
$$

see [20, Theorem 3.3.6].

Theorem 3.11. Let $A, B \in L(X)$ be such that $A B=B A$ and $\mathbf{T}:=\left(\begin{array}{cc}0 & A \\ B & 0\end{array}\right)$. Then we have:

(i) $A B$ has $S V E P$ if and only if $\mathbf{T}$ has $S V E P$.

(ii) $A B$ has property $(C)$ if and only if $\mathbf{T}$ has property $(C)$.

(iii) $A B$ has property $(\delta)$ if and only if $\mathbf{T}$ has property $(\delta)$.

(iv) $A B$ has property $(\beta)$ if and only if $\mathbf{T}$ has property $(\beta)$.

(v) $A B$ is a $H(p)$ operator if and only if $\mathbf{T}$ is a $H(p)$ operator.

Proof. (i) We have $\mathbf{T}^{2}==A B \oplus A B$. If $A B$ has SVEP then $\mathbf{T}^{2}$ has SVEP, see [2, Theorem 2.15] and this implies, by [2, Corollary 2.89], that $\mathbf{T}$ has SVEP. Conversely, if $\mathbf{T}$ has SVEP then $\mathbf{T}^{2}=A B \oplus A B$ has SVEP, always by [2, Corollary 2.89]. This implies that $A B$ has SVEP, again by [2, Theorem $2.15]$.

(ii) According Theorem 3.9, we know that $\mathbf{T}^{2}=A B \oplus A B$ has property $(C)$. Let $F$ be any closed subset of $\mathbb{C}$ and let $G$ be the set $\left\{z^{2}: z \in F\right\}$. Clearly, $G$ is closed, since the function $f(\lambda)=\lambda^{2}$ is continuous. If $X:=H \oplus H$, from the equality (12) we have

$$
\mathcal{X}_{\mathbf{T}^{2}}(G)=\mathcal{X}_{\mathbf{T}}\left(f^{-1}(G)\right)=\mathcal{X}_{\mathbf{T}}(F) .
$$

Since, by [20, Theorem 3.3.9], $\mathbf{T}^{2}$ has property $(C)$ then $\mathcal{X}_{\mathbf{T}^{2}}(G)$ is closed, and hence the glocal subspace $\mathcal{X}_{\mathbf{T}}(F)$ is closed, so $\mathbf{T}$ has property $(C)$. Conversely, 
if $\mathbf{T}$ has property $(C)$, then $\mathbf{T}^{2}=A B \oplus A B$ has property $(C)$, see $[20$, Theorem 3.3.6], and this easily implies that $A B$ has property $(C)$, since $\mathcal{X}_{\mathbf{T}^{2}}=$ $\mathcal{H}_{T}(A B) \oplus \mathcal{H}_{T}(A B)$.

(iii) $\mathbf{T}^{2}=A B \oplus A B$ has property $(\delta)$, byTheorem 3.9. Thus $\mathbf{T}$ has property $(\delta)$, by $[20$, Theorem 3.3.9]. Conversely, if $\mathbf{T}$ has property $(\delta)$, then $\mathbf{T}^{2}$, has property $(\delta)$, always by [20, Theorem 3.3.9], and hence $A B$ has the same property.

(iv) If $A B$ has property $(\beta)$ then its dual $(A B)^{\prime}$ has property $(\delta)$ and hence $(A B)^{*}=B^{*} A^{*}$ has property $(\delta)$. From part (iii) it the follows that $\mathbf{T}^{*}:=\left(\begin{array}{cc}0 & B^{*} \\ A^{*} & 0\end{array}\right)$ has property $(\delta)$ and hence $\mathbf{T}$ has property $(\beta)$. The reverse follows similarly.

(v) From $\mathbf{T}^{2}=A B \oplus A B$ we have $H_{0}\left(\lambda I-\mathbf{T}^{2}\right)=H_{0}(\lambda I-A B) \oplus$ $H_{0}(\lambda I-A B)$. If $A B$ is a $H(p)$-operator then $H_{0}(\lambda I-A B)=\operatorname{ker}(\lambda I-A B)^{p}$ for some $p \in \mathbb{N}$, so

$$
H_{0}\left(\lambda I-\mathbf{T}^{2}\right)=\operatorname{ker}(\lambda I-A B)^{p} \oplus \operatorname{ker}(\lambda I-A B)^{p}=\operatorname{ker}\left(\lambda I-\mathbf{T}^{2}\right)^{p},
$$

hence $\mathbf{T}^{2}$ has property $H(p)$. In $[22]$ it is shown that an operator $T$ has property $H(p)$ if and only if there exists an analytic function $f$ on a disc containing the spectrum such that $f(T)$ has property $H(p)$. This implies that also $\mathbf{T}$ has property $H(p)$. The converse follows in a similar way.

Corollary 3.12. Let $A, B \in L(H)$ be $J$-complex symmetric and suppose that $A B=B A$. If $\mathbf{T}:=\left(\begin{array}{cc}0 & A \\ B & 0\end{array}\right)$, then $\mathbf{T}$ is complex symmetric. If $A B$ has property $(\delta)$ then both $\mathbf{T}$ and $\mathbf{T}^{*}$ are decomposable and $H(p)$. Furthermore, all Weyl type theorems holds for $f(\mathbf{T})$ for every $f \in \mathcal{H}(\sigma(\mathbf{T}))$.

Proof. $\mathbf{J}$ is a conjugation for $\mathbf{T}$ and $\mathbf{T} \mathbf{J}=\mathbf{J} \mathbf{T}^{*}$, so $T$ is $\mathbf{J}$-complex symmetric. If $A B$ has property $(\delta)$ then $\mathbf{T}$ has property $(\delta)$, by Theorem 3.11 , and hence, by Theorem 3.2, $\mathbf{T}$ is decomposable. Furthermore, Weyl type theorems holds for $f(\mathbf{T})$ for every $f \in \mathcal{H}(\sigma(\mathbf{T})$.

Example 3.13. Let $T_{\phi}$ and $T_{\psi}$ be two Toeplitz operators, and consider the matrix operator $\mathbf{S}:=\left(\begin{array}{cc}0 & T_{\phi} \\ T_{\psi} & 0\end{array}\right)$, where $\phi, \psi \in H^{\infty}(\boldsymbol{\Gamma})$. We have $T_{\phi} T_{\psi}=T_{\phi \psi}$, see [7, Proposition 7.5], and since $\phi \psi \in H^{\infty}(\boldsymbol{\Gamma})$ then $T_{\phi \psi}$ is subnormal and hence, as observed in Example 3.7, has property $(\beta)$. Obviously, $T_{\phi}$ and $T_{\psi}$ commutes. By Theorem 3.11 then $\mathbf{S}$ has property $(\beta)$. Suppose now that $T_{\phi}$ and $T_{\psi}$ are complex symmetric with respect to the same conjugation $J$. Then $\mathbf{S}$ is $\mathbf{J}$-complex symmetric, so property $(\beta)$ for $\mathbf{S}$ is equivalent to property $(\delta)$. By Theorem 3.2, $\mathbf{S}$ satisfies all Weyl type theorems.

Example 3.14. If $g \in H^{2}(\mathbb{D})$, let $T_{g}$ denote the multiplication analytic Toeplitz operator by $g$. If $g, h \in H^{2}(\mathbb{D})$, consider the matrix $\mathbf{T}:=\left(\begin{array}{cc}0 & T_{g} \\ T_{h} & 0\end{array}\right)$. Both the operators $T_{g}, T_{h}$ have property $(\beta)$, see [20, Example 1.6.11], $T_{g} T_{h}=T_{h} T_{g}$ and also $T_{g} T_{h}=T_{g h}$ has property $(\beta)$. By Theorem 3.11 then $\mathbf{T}$ has property $(\beta)$. Observe that every multiplication analytic Toeplitz operator $T_{g}$ cannot 
have property $(\delta)$, see again [20, Example 1.6.11], hence $T_{g}$ cannot be complex symmetric, as already observed in Corollary 2.4 .

\section{Acknowledgements}

The authors wish to thank the referee for a careful reading and comments for the original draft.

Funding Open access funding provided by Universitá degli Studi di Palermo within the CRUI-CARE Agreement.

Open Access. This article is licensed under a Creative Commons Attribution 4.0 International License, which permits use, sharing, adaptation, distribution and reproduction in any medium or format, as long as you give appropriate credit to the original author(s) and the source, provide a link to the Creative Commons licence, and indicate if changes were made. The images or other third party material in this article are included in the article's Creative Commons licence, unless indicated otherwise in a credit line to the material. If material is not included in the article's Creative Commons licence and your intended use is not permitted by statutory regulation or exceeds the permitted use, you will need to obtain permission directly from the copyright holder. To view a copy of this licence, visit http:// creativecommons.org/licenses/by/4.0/.

Publisher's Note Springer Nature remains neutral with regard to jurisdictional claims in published maps and institutional affiliations.

\section{References}

[1] Aiena, P.: Algebraically paranormal operators on Banach spaces. Banach J. Math. Anal. 7(2), 136-145 (2013)

[2] Aiena, P.: Fredholm and Local Spectral Theory II, with Applications to Weyltype Theorems. Lecture Notes, vol. 2235. Springer, Berlin (2019)

[3] Aiena, P.: On the spectral mapping theorem for Weyl spectra of Toeplitz operators. Adv. Oper. Theory (2020). https://doi.org/10.1007/s43036-020-00069-3

[4] Aiena, P., Neumann, M.: On the stability of the localized single-valued extension property under commuting perturbations. Proc. Am. Math. Soc. 141(6), 2039-2050 (2013)

[5] Aiena, P., Colasante, M.L., Gonzalez, M.: Operators which have a closed quasinilpotent part. Proc. Am. Math. Soc. 130(9), 2701-2710 (2002)

[6] Conway, J.B.: The Theory of Subnormal Operators. Mathematical Surveys and Monographs, No 36. American Mathematical Society, Providence (1991)

[7] Douglas, R.G.: Banach Algebra Techniques in Operator Theory. Graduate Texts in Mathematics, vol. 179, 2nd edn. Springer, New York (1998)

[8] Duggal, B.P.: Upper triangular operator matrices, SVEP and Browder, Weyl's theorem. J. Math. Anal. Appl. 340, 366-373 (2008)

[9] Duggal, B.P.: Hereditarily polaroid operators, SVEP and Weyl's theorem. Integr. Equ. Oper. Th. 63, 17-28 (2009) 
[10] Dunford, N.: Spectral theory II. Resolution of the identity. Pac. J. Math. 2, 559-614 (1952)

[11] Dunford, N.: Spectral operators. Pac. J. Math. 4, 421-354 (1954)

[12] Garcia, S.R.: Conjugation and Clark operators. Contemp. Math. 393, 67-112 (2006)

[13] Garcia, S.R., Wogen, W.R.: Some new classes of complex symmetric operators. Trans. Am. Math. Soc. 362, 6065-6077 (2010)

[14] Guo, K., Zhu, S.: A canonical decomposition for complex symmetric operators. J. Oper. Theory 72, 529-547 (2014)

[15] Jung, S., Lee, M., Lee, J.: On local spectral properties of complex symmetric operators. J. Math. Anal. Appl. 379, 325-333 (2011)

[16] Jung, S., Lee, M., Lee, J.: Properties of complex symmetric operators. Oper. Matrices 8, 325-333 (2011)

[17] Jung, S., Lee, M., Lee, J.: On scalar extensions and spectral decompositions of complex symmetric operators. J. Math. Anal. Appl. 384, 252-260 (2011)

[18] Jung, S., Ko, E., Lee, J.: On complex symmetric operator matrices. J. Math. Anal. Appl. 406, 373-385 (2013)

[19] Ko, E., Lee, J.: On complex symmetric Toeplitz operators. J. Math. Anal. Appl. 434, 229-547 (2016)

[20] Laursen, K.B., Neumann, M.M.: An Introduction to Local Spectral Theory. Clarendon Press, Oxford (2000)

[21] Miller, T.L., Miller, V.G., Neumann, M.M.: Operators with closed analytic core. Rend. Circolo Mat. Palermo. 51(3), 495-502 (2003)

[22] Oudghiri, M.: Weyl's and Browder's theorem for operators satisfying SVEP. Stud. Math. 163(1), 85-101 (2004)

[23] Smith, R.C.: Local spectral theory for invertible composition operators on $H^{p}$. Integr. Equ. Oper. Th. 25, 329-35 (1996)

[24] Wang, X., Gao, Z.: A note on Aluthge transforms of complex symmetric operators and applications. Integr. Equ. Oper. Th. 65, 573-580 (2009)

Pietro Aiena, Fabio Burderi and Salvatore Triolo

Dipartimento d'Ingegneria

Università di Palermo

Viale delle Scienze

90128 Palermo

Italy

e-mail: pietro.aiena@unipa.it

Fabio Burderi

e-mail: fabio.burderi@unipa.it

Salvatore Triolo

e-mail: salvatore.triolo@unipa.it

Received: June 7, 2020.

Revised: November 24, 2020.

Accepted: February 10, 2021. 